\title{
Radiation enhances the invasion abilities of pulmonary adenocarcinoma cells via STAT3
}

\author{
FENGSHENG LI ${ }^{1,2^{*}}$, LING GAO $^{3 *}$, ZHIDONG WANG ${ }^{1}$, BO DONG ${ }^{1}$, \\ TAO YAN $^{2}$, QISHENG JIANG ${ }^{2}$ and XIAOHUA CHEN ${ }^{1}$
}

\author{
${ }^{1}$ Department of Experimental Therapy of ARS, Beijing Institute of Radiation Medicine, Beijing 100850; \\ ${ }^{2}$ The Second Artillery General Hospital, Beijing 100088; ${ }^{3}$ Key Laboratory of Radiological Protection and Nuclear Emergency, \\ National Institute for Radiological Protection, Chinese Center for Disease Control and Prevention, Beijing 100088, P.R. China
}

Received December 18, 2012; Accepted April 12, 2013

DOI: $10.3892 / \mathrm{mmr} .2013 .1441$

\begin{abstract}
In the present study, the effect of radiation on the invasion of the pulmonary adenocarcinoma cell line, A549, was investigated. Invasion of A549 cells irradiated with 2 and 4 Gy doses of $\gamma$-ray was detected using the transwell Matrigel invasion assay. Levels of matrix metalloproteinase 2 (MMP-2) and phosphorylated signal transducer and activator of transcription 3 (STAT3) were detected by reverse transcription PCR (RT-PCR) and/or immunoblotting. The enzyme activity of MMP-2 was examined by gelatin zymography. Results demonstrated that the invasion of A549 cells was significantly enhanced by $\gamma$-ray radiation at doses of 2 or $4 \mathrm{~Gy}$. In addition, exposure to radiation was found to promote transcriptional expression of MMP-2 and increase MMP-2 enzyme activity. Irradiation activated the phosphorylation of STAT3 and promoted the nuclear localization of STAT3. The blockage of STAT3 phosphorylation using a specific inhibitor (AG490) suppressed the irradiation-induced elevation of MMP-2 expression, enzyme activity and invasion of A549 cells. Finally, the expression of vascular endothelial growth factor (VEGF) was found to be upregulated by radiation, which was associated with the activation of STAT3. Results of the current study indicate that irradiation leads to activation of STAT3 and translocation to the nucleus, leading to activation of VEGF and MMP-2 transcription, resulting in the increased invasion of A549 cells.
\end{abstract}

Correspondence to: Professor Xiaohua Chen, Department of Experimental Therapy of ARS, Beijing Institute of Radiation Medicine, Taiping Road 27, Beijing 100850, P.R. China

E-mail: xiaohuachen856@yahoo.cn

Dr Qisheng Jiang, The Second Artillery General Hospital, Xinwai street 16, Xicheng District, Beijing 100088, P.R. China

E-mail: jqs598@sina.com

*Contributed equally

Key words: radiation, invasion, lung cancer, signal transducer and activator of transcription 3 , matrix metalloproteinase

\section{Introduction}

Radiation therapy is important for the treatment of malignant tumors, with $\sim 60 \%$ of cancer patients requiring radiotherapy as a component of their cancer treatment (1). However, since it is genotoxic, radiation also activates various radiation response genes. Activation of these genes leads to the production of cytokines that are able to alter the microenvironment surrounding tumor cells, which in turn increases the invasion and metastasis of surviving tumor cells $(2,3)$. Previous studies have demonstrated that radiation promotes the invasion or metastasis of various types of cancer cells, including pancreatic, glioma, hepatocellular, breast and melanoma (4-7). In addition, a clinical study revealed that invasive recurrence increased following treatment of ductal carcinoma in situ with radiation therapy $(8)$.

Cancer cell invasion and metastasis are complicated processes involving cell proliferation at the primary site, adhesion to and invasion of basement membranes, migration through the extracellular matrix (ECM), penetration into lymphatic or blood vessels and angiogenesis at the metastatic site (9). During the invasion of basement membranes by cancer cells, matrix metalloproteinases (MMPs), members of the matrixin subfamily of the zinc metalloproteinases, play key roles in degradation of the ECM (10), and elevated levels of MMPs have been found in a number of tumor types (11). MMP-2 degrades denatured interstitial collagens I and III, as well as native collagen IV, which is an important component of the basement membrane. Activated MMP-2 triggers tumor invasion and metastasis and increased expression of MMP-2 is associated with poor prognosis in cancer patients (12). Notably, the expression and activity of MMP-2 is enhanced by radiation in a number of cells, including astrocytes and mesangial and kidney tubule epithelial cells in rats and bronchial epithelial cells in humans (13-16). However, the biological function of elevated MMP-2 expression induced by radiation remains unclear.

The transcription factor signal transducer and activator of transcription 3 (STAT3), a member of the STAT family, plays a critical role in mediating cell survival, proliferation, invasion and angiogenesis (17). STAT3 includes a conserved tyrosine residue at position 705 (Tyr705), which is phosphorylated 
by Janus-activated kinase 2 (18). STAT3 phosphorylation promotes its dissociation from the receptor and its homodimerization. The dimer then translocates to the nucleus, where it regulates the transcription of target genes (19), including MMP-2 and MMP-9. Blocking STAT3 signaling in highly metastatic mouse melanoma cells was reported to significantly inhibit the invasion of tumor cells by suppressing expression of the MMP-2 gene (20). Inhibition of STAT3 also prevents the metastasis of breast cancer cells (21). Collectively, previous studies have indicated that STAT3 affects tumor invasion, growth and metastasis.

E-cadherin is important for cell-to-cell cohesion, cell-to-cell recognition and epithelial polarity (22), and analysis of E-cadherin protein expression levels is an important indicator of cell invasion ability.

Despite the therapeutic role of radiation in lung cancer, particularly in non-small cell lung cancer (NSCLC), radiation also promotes the invasion of NSCLC (23). NSCLC invasion is associated with poor prognosis since it increases the invasive growth and metastasis of tumors; for example, in mouse Lewis lung cancer (24).

In the current study, the hypothesis that radiation promotes the invasion of lung adenocarcinoma A549 cells was investigated. In addition, the effect of radiation on the secretion of MMP-2 in A549 cells and the molecular mechanism by which A549 cell invasion is induced by radiation was determined. Radiation was found to promote the invasion of A549 cells and the secretion of MMP-2, which may represent the molecular mechanism of radiation-induced A549 cell invasion. The previous observation that STAT3 plays a key role in radiation-induced A549 cell invasion was further confirmed in this study.

\section{Materials and methods}

Cell lines. The A549 cell line was obtained from the Cell Culture Center of The Institute of Basic Medical Sciences (Chinese Academy of Medical Sciences, Beijing, China). Cells were maintained in Dulbecco's Modified Eagle's Medium (DMEM) plus $10 \%$ fetal bovine serum at $37^{\circ} \mathrm{C}$ within humidified $5 \% \mathrm{CO}_{2}$ air. The present study was approved by the Ethics committee of The Beijing Institute of Radiation Medicine, Beijing, China.

Radiation and chemical reagents. A549 cells were irradiated using a Cobalt-60 unit (Beijing Institute of Radiation Medicine, Beijing, China) at a source-skin distance of $4 \mathrm{~m}$. The dose rate was $2.17 \mathrm{~Gy} / \mathrm{min}$. The chemical inhibitor, AG490 (Sigma-Aldrich, St. Louis, MO, USA), was used to suppress the radiation-induced invasion of A549 cells.

Invasion assay. The invasion of A549 cells was measured as the number of cells invading through Matrigel-coated transwell inserts, as described previously (25). In brief, transwell inserts with $8-\mu \mathrm{m}$ pores were coated with Matrigel (20 $\mu \mathrm{g} /$ well; BD Biosciences, Franklin Lakes, NJ, USA). A total of $2 \times 10^{5}$ cells were suspended in $200 \mu 1$ serum-free DMEM and seeded into the upper chamber. DMEM supplemented with $10 \%$ fetal bovine serum (FBS) was placed in the lower chamber as a chemoattractant. Cells were allowed to invade for $20 \mathrm{~h}$. Following incubation, non-invaded cells on the upper side of the membrane were removed using a cotton swab. The invasive cells on the lower surface of the Matrigel-coated membrane were fixed with $100 \%$ methanol and stained using Wright's stain (Sigma-Aldrich). Invasion was determined by counting cells in 5 microscopic fields/well and the extent of invasion was expressed as an average number of cells/microscopic field.

Cell adhesion assay. Firstly, 96-well plates were coated with $10 \mu \mathrm{g} / \mathrm{ml}$ fibronectin in phosphate buffered saline (PBS; both Sigma-Aldrich) overnight at $4^{\circ} \mathrm{C}$. Wells were then rinsed and blocked with $1 \%$ bovine serum albumin in PBS for $1 \mathrm{~h}$. Cells were harvested during the logarithmic phase of growth by trypsin and plated at $5 \times 10^{4}$ cells/well. Following $1 \mathrm{~h}$ incubation at $37^{\circ} \mathrm{C}$, wells were rinsed to remove non-adherent cells. Adhered cells were treated with $100 \mu 10.05 \%$ 3-(4,5-Dimethyl-2-thiazolyl)-2,5-diphenyl-2H-tetrazolium bromide (MTT) dissolved in PBS and incubated for $1 \mathrm{~h}$. Following incubation, the MTT solution was removed and formazan formed by the cells was resuspended in $100 \%$ ethanol. Absorbance was measured at $590 \mathrm{~nm}$ using a Versamax microplate reader (Amersham Pharmacia Biotech, Amersham, UK).

Western blot analysis. Cells were harvested 12 or $24 \mathrm{~h}$ following irradiation and were then directly lysed in a lysis buffer containing a mammalian protease cocktail to obtain total protein content. For nuclear extracts, cells were lysed in a NE-PER extraction reagent (Pierce Biotechnology, Inc., Rockford, IL, USA), according to the manufacturer's instructions. Protein concentration was measured using the Bradford method. Protein $(50 \mu \mathrm{g})$ was separated on a $12 \%$ sodium dodecyl sulfate-polyacrylamide gel and transferred onto nitrocellulose membranes. Membranes were blocked in TBST buffer (10 mM Tris-HCl, $\mathrm{pH} 8.0 ; 0.15 \mathrm{M}$ $\mathrm{NaCl}$; and $0.05 \%$ Tween 20 ) containing $5 \%$ skimmed milk. Next, membranes were incubated overnight with monoclonal antibodies against p-STAT3 (Y705; Cell Signaling Technology, Inc., Danvers, MA, USA), MMP-2 (Santa Cruz Biotechnology, Inc., Santa Cruz, CA, USA), vascular endothelial growth factor (VEGF; Sigma-Aldrich), $\beta$-actin (Santa Cruz Biotechnology, Inc.) and E-cadherin (clone ECH-6; Cell Marque Corp., Rocklin, CA, USA). Finally, horseradish peroxidase-linked anti-mouse IgG (Zhongshan Goldenbridge Biotechnology Co., Beijing, China) was added to allow enhanced chemiluminescence visualization of the bands.

Gelatin zymography. Subconfluent A549 cells (70-80\% confluency) were washed and refreshed with serum-free DMEM, and then irradiated and incubated for $24 \mathrm{~h}$. The activity of electrophoretically separated gelatinolytic enzymes in the conditioned medium of A549 cells was determined by gelatin zymography, as described previously (24). Zones of gelatinolytic activity were detected as clear bands against a blue background.

Reverse transcription-PCR (RT-PCR). Total cellular RNA content was extracted with TRIzol (Gibco-BRL, Carlsbad, CA, USA), according to the manufacturer's instructions. Complementary first-strand DNA was generated using an ImProm-II reverse transcription system (Promega, Shanghai, 

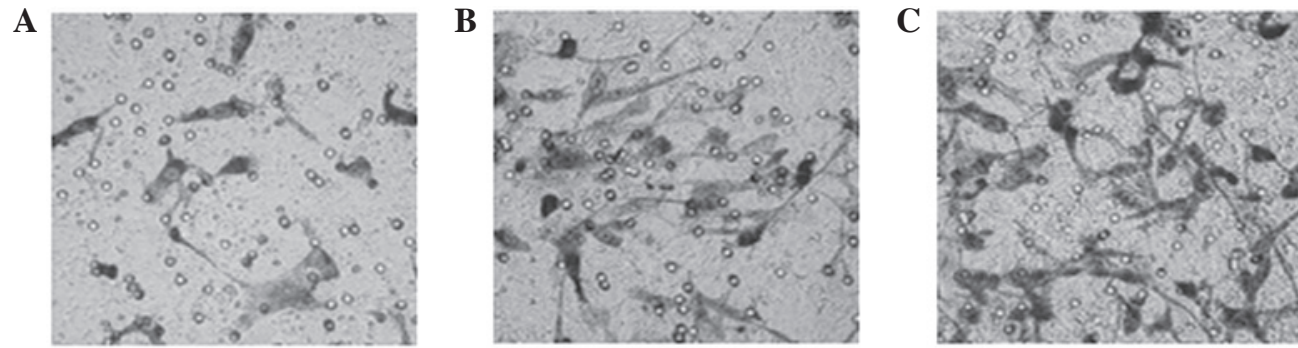

Figure 1. Radiation enhances the invasion of A549 cells after 20 h. Cells irradiated with doses of (A) 0 , (B) 2 and (C) 4 Gy
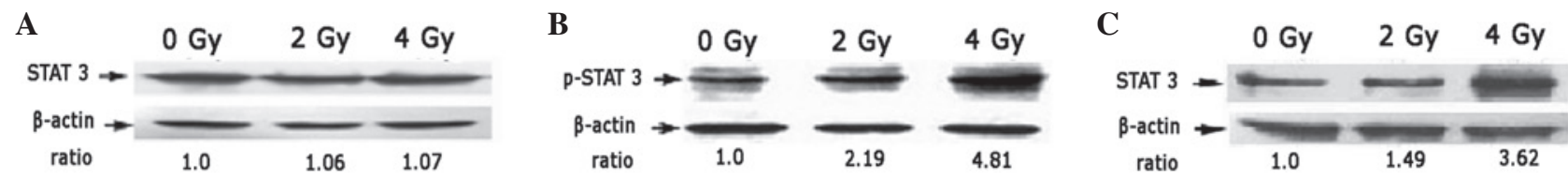

Figure 2. Radiation activates the phosphorylation and nucleic localization of STAT3. Protein expression levels of (A) STAT3, (B) phosphorylated STAT3 and (c) nuclear STAT3. STAT, signal transducer and activator of transcription 3.
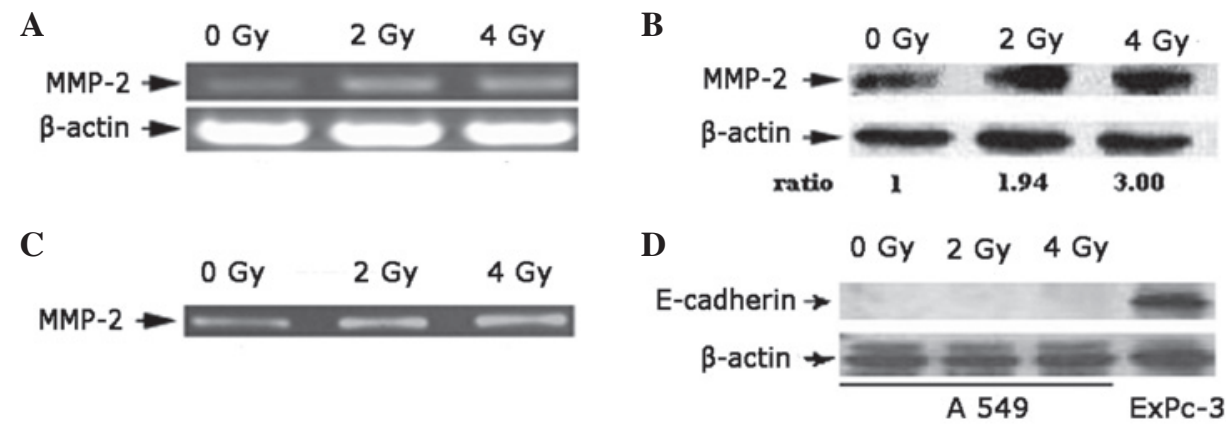

Figure 3. Radiation increases MMP-2 mRNA expression of A549 cells after 24 h (0, 2 and 4 Gy). (A) Total RNA expression following RT-PCR analysis (B) Western blot analysis of MMP-2 expression. (C) Gelatin zymography analysis of MMP-2 enzyme activity. (D) Western blot analysis of E-cadherin expression. MMP-2, matrix metalloproteinase 2; RT-PCR, reverse transcription PCR.

China), according to the manufacturer's instructions. The PCR protocol involved an initial denaturation step at $94^{\circ} \mathrm{C}$ for $5 \mathrm{~min}$, followed by 30 cycles at $94^{\circ} \mathrm{C}$ for $30 \mathrm{sec}, 57^{\circ} \mathrm{C}$ for $30 \mathrm{sec}$ and $72^{\circ} \mathrm{C}$ for $30 \mathrm{sec}$. Oligonucleotide primer sequences were as follows: MMP-2, 5'-GGCCAAGTGGTCCGTGTG-3' (sense) and 5'-GAGGCCCCATAGAGCTCC-3' (antisense); and $\beta$-actin, 5'-CATCTCTTGCTCGAAGTCCA-3' (sense) and 5'-ATCATGTTTGAGACCTTCAACA-3' (antisense). PCR was performed using a PCR Mix kit [Tiangen Biotech (Beijing) Co., Ltd., Beijing, China], according to the manufacturer's instructions.

Statistical analysis. All experiments were performed in triplicate or quadruplicate and 3 measurements/data point were obtained in each experiment. A two-tailed Student's t-test was used for statistical analysis of comparative data using SPSS software (SPSS, Inc., Chicago, IL, USA). P<0.05 was considered to indicate a statistically significant difference.

\section{Results}

Radiation promotes the invasion of A549 cells but has no effect on cell adhesion. Radiation doses of 2 or 4 Gy were found to significantly promote the invasion of A549 cells in a dose-dependent manner compared with the untreated controls (Fig. 1). Cell adhesion, another important factor that affects tumor metastasis, was unchanged by radiation.

Radiation induces STAT3 activation and promotes its nuclear localization. The expression of STAT3 in A549 cells was not affected by 2 or 4 Gy radiation (Fig. 2A). However, levels of phosphorylated STAT3, the functional form of STAT3, in A549 cells were observed to increase significantly $12 \mathrm{~h}$ after 2 or $4 \mathrm{~Gy}$ radiation compared with the untreated controls (Fig. 2B). These observations indicate that 2 and 4 Gy radiation induces the activation of STAT3. Total STAT3 in the nucleus of A549 cells irradiated with 2 or 4 Gy radiation was identified to be significantly higher than in untreated cells (Fig. 2C).

Radiation increases the expression and activity of MMP-2. RT-PCR results indicate that the expression of MMP-2 mRNA in A549 cells increased significantly $24 \mathrm{~h}$ after 2 or 4 Gy radiation compared with the untreated controls (Fig. 3A). An upregulated expression of MMP-2 protein in A549 cells $24 \mathrm{~h}$ after radiation was also detected (Fig. 3B), and the expression of MMP-2 protein at 2 Gy was higher than levels at 4 Gy. The activity 

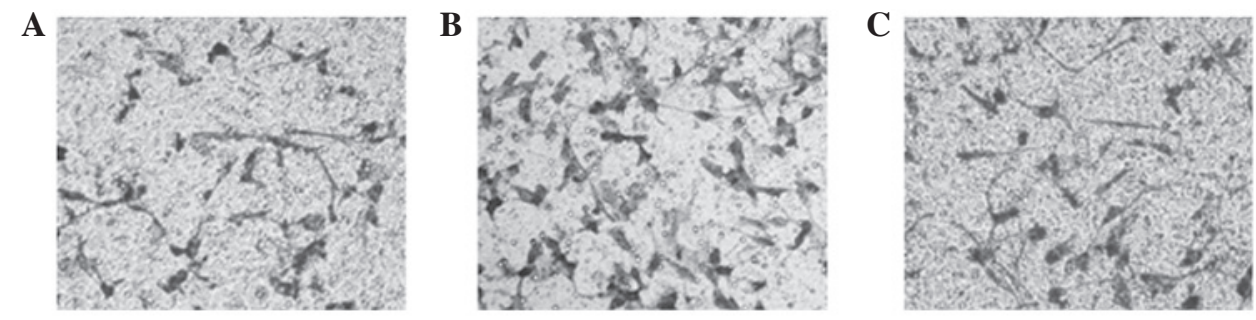

Figure 4. Radiation-induced secretion of MMP-2 and the invasion of A549 cells is blocked by AG490. (A) Untreated; (B) irradiated with 4 Gy; and (C) irradiated with 4 Gy and treated with $20 \mu \mathrm{M}$ AG490. MMP-2, matrix metalloproteinase 2.

A

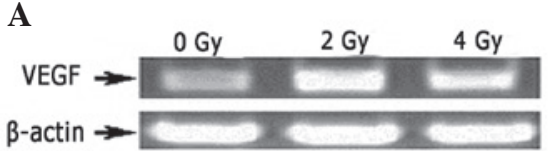

B

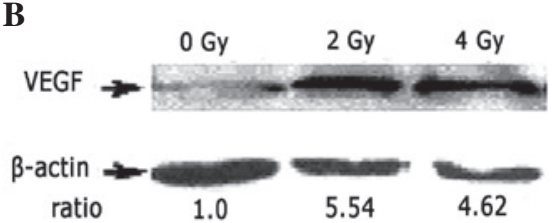

C

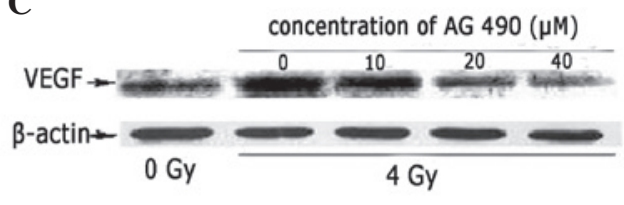

Figure 5. Radiation induces VEGF expression in A549 cells after 24 h. (A) RT-PCR analysis of VEGF mRNA expression at various doses of irradition. Western blot analysis of VEGF protein expression following (B) irradiation $(0,2$ or $4 \mathrm{~Gy})$ and $(\mathrm{C})$ irradiation $(0$ or $4 \mathrm{~Gy})$ followed by treatment with various concentrations of AG490. $\beta$-actin was used as control. VEGF, vascular endothelial growth factor; RT-PCR, reverse transcription PCR.

of the MMP-2 enzyme in the conditioned medium increased following the irradiation of A549 cells (Fig. 3C). The expression of E-cadherin was not affected by radiation (Fig. 3D), consistent with results of the cell adhesion assay. E-cadherin protein was not detected in A549 cells prior to and following irradiation.

Inhibition of STAT3 phosphorylation suppresses the radiation-induced expression of MMP-2 and invasion of A549 cells. The increase in radiation-induced MMP-2 expression triggered by 4 Gy radiation was suppressed when A549 cells were treated with 20 or $40 \mu \mathrm{M}$ AG490 (Fig. 4A). In addition, AG490 inhibited the enzymatic activity of MMP-2 (Fig. 4B). Since radiation-induced activation of STAT3 in A549 cells leads to the elevated expression of MMP-2, we investigated whether inhibition of STAT3 eliminates the increased invasion of A549 cells. Results demonstrate that $20 \mu \mathrm{M}$ AG490 suppressed the radiation-induced invasion of A549 cells (Fig. 4C).

Radiation increases the expression of VEGF via activation of STAT3. Doses of 2 or 4 Gy irradiation were found to significantly increase the transcription of VEGF, indicating that irradiation regulated VEGF expression at the transcriptional level (Fig. 5A and B). Further immunoblotting assays revealed that VEGF protein expression was also significantly elevated by 2 or $4 \mathrm{~Gy}$ irradiation. The radiation-induced elevation of VEGF (Fig. 5C) was significantly suppressed by AG490, consistent with MMP-2 expression.

\section{Discussion}

Lung cancer is the leading cause of cancer mortality worldwide and radiotherapy is the primary mode of treatment, particularly in cases of non-resectable lung cancer. Although radiotherapy generally increases survival in lung cancer patients, the majority of patients are likely to suffer from metastases in distant organs. In the current study, irradiation was found to enhance A549 cell invasion, consistent with the observation that radiotherapy of the primary tumor is associated with unpredictable metastatic effects (24). In addition, a previous in vivo study revealed that irradiation promoted the metastatic growth of Lewis lung cancer, a type of mouse pulmonary adenocarcinoma (26). In the present study, increased irradiation-induced invasion of A549 cells was, at least in part, mediated by increased MMP-2 expression. Additional analyses demonstrated that the activation of STAT3 was involved in irradiation-induced MMP-2 expression and activation, ultimately promoting the invasion of A549 cells. Notably, the activation of STAT3 by irradiation also increased the expression of VEGF in A549 cells.

The malignant characteristics of tumor cells include rapid cell growth, migration, invasion and metastasis. Metastasis itself is the most insidious and life-threatening property of cancer cells, and is preceded by migration and invasion. In a previous study, it was demonstrated that ionizing irradiation increases A549 cell migration (27). Results of the present study were consistent with these observations, whereby the invasive ability of A549 cells was also enhanced following 2 and 4 Gy irradiation. In addition, VEGF expression was found to be significantly increased in irradiated A549 cells. Since specific radioresistant tumor cells are able to survive radiation therapy, these results indicated that irradiation therapy may increase tumor metastasis due to the increased migration, invasion and pro-angiogenic ability of surviving cells.

MMPs degrade and modify the ECM, facilitating cancer cell invasion, and have been reported to be involved in mammalian tumor progression and metastasis. Altered expression of MMPs is a recognized hallmark of invasive tumor cells (28), and MMPs have been found to be upregulated in cancer cells. Previous studies have revealed that irradiation promotes MMP-9 expression in hepatocellular carcinoma cells (29) and MMP-2 expression in glioma cells (30), and elevated expression levels of MMP-2 or MMP-9 have been revealed to promote irradiation-induced cancer cell invasion. Similarly, results of the present study demonstrated that MMP-2 is also 
upregulated in pulmonary adenocarcinoma cells following irradiation. Radisky and Radisky (31) previously demonstrated that MMP-2 promotes and mediates epithelial-mesenchymal transition (EMT), a process implicated in tumor progression and promotion of the migration and invasion of cancer cells Notably, irradiation-induced EMT-associated changes in A549 were observed in a previous study (27). Combined with observations of the present study, these results indicate that irradiation-induced increases in MMP-2 regulated the invasion of A549 cells by promoting EMT-associated changes, as well as by having a direct pro-invasive activity.

STAT3, which has been found to be phosphorylated in several clinical cancer cells, is activated by numerous cytokines, growth factors and oncogenic proteins (32). In the current study, STAT3 was also found to be activated by irradiation. Similarly, Singh-Gupta et al (33) found that irradiation promoted the phosphorylation of STAT3 in prostate cancer cells.

Abdulghani et al (34) reported that STAT3 is associated with metastatic progression in various types of cancer, including lung, skin, liver, ovarian, kidney, colon and prostate cancer. Activation of STAT3 may activate the expression of genes associated with the regulation of cell migration, invasion and angiogenesis at the transcriptional level. The expression of these genes, including MMPs and VEGF, promote tumor metastasis. In the current study, irradiation-induced STAT3 activation was identified to be involved in the upregulation of MMP-2, which facilitated the invasion of A549 cells. In addition, the activation of STAT3 was involved in irradiation-induced VEGF expression, indicating that STAT3 activation mediates tumor metastasis by several mechanisms.

In conclusion, in the present study, $\gamma$-ray radiation was found to enhance A549 cell invasion, largely via the upregulation of MMP-2 expression mediated by STAT3. The ability of irradiation to kill tumor cells is definitive and has been demonstrated in a number of in vitro and in vivo studies (35-37). However, at present, only a limited number of studies on the effect of irradiation on tumor cell invasion have been performed. Based on the widespread application of radiotherapy for lung cancer and the poor outcomes associated with increased invasion, results of the present study indicate the importance of suppression of the irradiation-induced invasion of lung cancer cells. Identification of the key mediator involved in this process is likely to lead to development of a specific inhibitor to block metastatic signaling, while retaining the therapeutic benefit of irradiation for lung cancer therapy.

\section{Acknowledgements}

The present study was supported by Science Funds for Young Scholar of Chinese Center for Disease Control and Prevention (nos. 2011A202), and grants from the National Natural Science Foundation of China (nos. 81001216, 81172130 and 81202151).

\section{References}

1. Klepper L: An interactive method for optimization of irradiation plans in radiation therapy of malignant tumors. Med Tekh: 25-30, 2006 (In Russian).

2. Rastogi S, Boylan M, Wright EG and Coates PJ: Interactions of apoptotic cells with macrophages in radiation-induced bystander signaling. Radiat Res 179: 135-145, 2013.
3. Sun Y and Nelson PS: Molecular pathways: involving microenvironment damage responses in cancer therapy resistance. Clin Cancer Res 18: 4019-4025, 2012.

4. Madani I, De Neve W and Mareel M: Does ionizing radiation stimulate cancer invasion and metastasis? Bull Cancer 95: 292-300, 2008.

5. Kaur H, Buettner H, Salomao DR and Marks RS: Transcleral orbital invasion by a radiation and chemotherapy-resistant choroidal metastasis of a pulmonary adenocarcinoma. Am J Ophthalmol 143: 369-370, 2007.

6. Itoh YH, Nakatsugawa S, Oguri T and Miyata N: Increase of cellular fibrinolysis in human lung cancer cell line by radiation: relationship between urokinase-type plasminogen activator (uPA) and metastasis and invasion. Nihon Igaku Hoshasen Gakkai Zasshi 56: 747-749, 1996 (In Japanese).

7. Boutrus R, Abi-Raad R, Niemierko A, et al: Does lymphovascular invasion predict regional nodal failure in breast cancer patients with zero to three positive lymph nodes treated with conserving surgery and radiotherapy? Implications for regional radiation. Int J Radiat Oncol Biol Phys 78: 793-798, 2010.

8. Guerra LE, Smith RM, Kaminski A, Lagios MD and Silverstein MJ: Invasive local recurrence increased after radiation therapy for ductal carcinoma in situ. Am J Surg 196: $552-555,2008$

9. Fidler IJ, Kim SJ and Langley RR: The role of the organ microenvironment in the biology and therapy of cancer metastasis. J Cell Biochem 101: 927-936, 2007.

10. Kargozaran H, Yuan SY, Breslin JW, et al: A role for endothelial-derived matrix metalloproteinase-2 in breast cancer cell transmigration across the endothelial-basement membrane barrier. Clin Exp Metastasis 24: 495-502, 2007.

11. Kerkelä E and Saarialho-Kere U: Matrix metalloproteinases in tumor progression: focus on basal and squamous cell skin cancer. Exp Dermatol 12: 109-125, 2003.

12. Stetler-Stevenson WG: The role of matrix metalloproteinases in tumor invasion, metastasis, and angiogenesis. Surg Oncol Clin N Am 10: 383-392, 2001.

13. Araya J, Maruyama M, Sassa K, et al: Ionizing radiation enhances matrix metalloproteinase-2 production in human lung epithelial cells. Am J Physiol Lung Cell Mol Physiol 280: L30-L38, 2001.

14. Sawaya R, Tofilon PJ, Mohanam S, et al: Induction of tissue-type plasminogen activator and 72-kDa type-IV collagenase by ionizing radiation in rat astrocytes. Int J Cancer 56: 214-218, 1994.

15. Zhao W, O'Malley Y and Robbins ME: Irradiation of rat mesangial cells alters the expression of gene products associated with the development of renal fibrosis. Radiat Res 152: 160-169, 1999.

16. Zhao W, O'Malley Y, Wei S and Robbins ME: Irradiation of rat tubule epithelial cells alters the expression of gene products associated with the synthesis and degradation of extracellular matrix. Int J Radiat Biol 76: 391-402, 2000.

17. Lui VW, Boehm AL, Koppikar P, et al: Antiproliferative mechanisms of a transcription factor decoy targeting signal transducer and activator of transcription (STAT) 3: the role of STAT1. Mol Pharmacol 71: 1435-1443, 2007.

18. Ju H, Venema VJ, Liang H, Harris MB, Zou R and Venema RC: Bradykinin activates the Janus-activated kinase/signal transducers and activators of transcription (JAK/STAT) pathway in vascular endothelial cells: localization of JAK/STAT signalling proteins in plasmalemmal caveolae. Biochem J 351: 257-264, 2000.

19. Shen Y, Devgan G, Darnell JE Jr and Bromberg JF: Constitutively activated Stat 3 protects fibroblasts from serum withdrawal and UV-induced apoptosis and antagonizes the proapoptotic effects of activated Stat1. Proc Natl Acad Sci USA 98: 1543-1548, 2001.

20. Li Q, Wang Y, Xie C, Qiu X and Wang E: Expression of MMP-2, MMP-9, TIMP-1 and their relationship with prognosis in NSCLC. Zhongguo Fei Ai Za Zhi 7: 497-500, 2004 (In Chinese).

21. Gariboldi MB, Ravizza R, Molteni R, Osella D, Gabano E and Monti E: Inhibition of Stat 3 increases doxorubicin sensitivity in a human metastatic breast cancer cell line. Cancer Lett 258: 181-188, 2007.

22. Jeanes A, Gottardi CJ and Yap AS: Cadherins and cancer: how does cadherin dysfunction promote tumor progression? Oncogene 27: 6920-6929, 2008.

23. Tsutsumi K, Tsuda M, Yazawa N, et al: Increased motility and invasiveness in tumor cells that survive $10 \mathrm{~Gy}$ irradiation. Cell Struct Funct 34: 89-96, 2009. 
24. Camphausen K, Moses MA, Beecken WD, Khan MK, Folkman J and O'Reilly MS: Radiation therapy to a primary tumor accelerates metastatic growth in mice. Cancer Res 61: 2207-2211, 2001.

25. Zhang W, Nwagwu C, Le DM, Yong VW, Song H and Couldwell WT: Increased invasive capacity of connexin43-overexpressing malignant glioma cells. J Neurosurg 99: 1039-1046, 2003.

26. Mayo JG: Biologic characterization of the subcutaneously implanted Lewis lung tumor. Cancer Chemother Rep 2 3: 325-330, 1972.

27. Jung JW, Hwang SY, Hwang JS, Oh ES, Park S and Han IO: Ionising radiation induces changes associated with epithelial-mesenchymal transdifferentiation and increased cell motility of A549 lung epithelial cells. Eur J Cancer 43: 1214-1224, 2007.

28. Béliveau A, Bérubé M, Rousseau A, Pelletier G and Guérin SL: Expression of integrin alpha5betal and MMPs associated with epithelioid morphology and malignancy of uveal melanoma. Invest Ophthalmol Vis Sci 41: 2363-2372, 2000

29. Cheng JC, Chou CH, Kuo ML and Hsieh CY: Radiation-enhanced hepatocellular carcinoma cell invasion with MMP-9 expression through PI3K/Akt/NF-kappaB signal transduction pathway. Oncogene 25: 7009-7018, 2006.

30. Park CM, Park MJ, Kwak HJ, et al: Ionizing radiation enhances matrix metalloproteinase-2 secretion and invasion of glioma cells through Src/epidermal growth factor receptor-mediated p38/Akt and phosphatidylinositol 3-kinase/Akt signaling pathways. Cancer Res 66: 8511-8519, 2006.
31. Radisky ES and Radisky DC: Matrix metalloproteinase-induced epithelial-mesenchymal transition in breast cancer. J Mammary Gland Biol Neoplasia 15: 201-212, 2010.

32. Devarajan E and Huang S: STAT3 as a central regulator of tumor metastases. Curr Mol Med 9: 626-633, 2009.

33. Singh-Gupta V, Zhang H, Banerjee S, et al: Radiation-induced HIF-1alpha cell survival pathway is inhibited by soy isoflavones in prostate cancer cells. Int J Cancer 124: 1675-1684, 2009.

34. Abdulghani J, Gu L, Dagvadorj A, et al: Stat3 promotes metastatic progression of prostate cancer. Am J Pathol 172: 1717-1728, 2008.

35. Lynch DH, Gurish MF and Daynes RA: The effects of ultraviolet irradiation on the generation of anti-tumor cytotoxic effector cell responses in vitro. J Immunol 127: 1163-1168, 1981.

36. Frey B, Rubner Y, Wunderlich R, et al: Induction of abscopal anti-tumor immunity and immunogenic tumor cell death by ionizing irradiation - implications for cancer therapies. Curr Med Chem 19: 1751-1764, 2012.

37. Jürgenliemk-Schulz IM, Renes IB, Rutgers DH, et al: Anti-tumor effects of local irradiation in combination with peritumoral administration of low doses of recombinant interleukin-2 (rIL-2). Radiat Oncol Investig 5: 54-61, 1997. 